\title{
Evaluación del manejo de residuos sólidos en la Universidad Pontificia Bolivariana seccional Bucaramanga
}

\author{
Solid Waste Management Evaluation at the Universidad Pontificia \\ Bolivariana, Bucaramanga's Campus
}

Fecha de Recepción: 2013-05-13

Fecha de Aprobación: 2013-06-28
Luis Eduardo Castillo Meza*

Marianela Luzardo Briceño**

\section{Resumen}

La evaluación del manejo de residuos sólidos en la Universidad Pontificia Bolivariana seccional Bucaramanga se desarrolló en cuatro etapas: caracterización de los residuos sólidos que van a disposición final, cuantificación de residuos destinados a aprovechamiento, evaluación de otros factores asociados a la gestión de los residuos sólidos y el análisis estadístico de la información. Se encontró que el residuo más generado es comida y jardinería, con una participación del $37 \%$, equivalente a 149 $\mathrm{kg}$ /día. Se identificaron buenas prácticas de aprovechamiento para papel, cartón, metales y comida compostable. Por otra parte, para el año 2012 se estableció una producción per cápita de $0,052 \mathrm{~kg} /$ persona.día. Para el análisis estadístico

\section{Abstract}

An evaluation of the solid waste management process at the Universidad Pontificia Bolivariana -Bucaramanga, Campus-. Was performed through four stages: Characterization of the solid waste to be disposed, quantifying the recyclable or reusable solid waste, evaluation of additional factors associated with the solid waste management process and the statistical analysis of the gathered information. Results show that food and gardening waste generation was predominant, with $149 \mathrm{~kg} /$ day, corresponding to $37 \%$ of all solid waste. Good practices for reuse and recycle of paper, cardboard, metals and compostable food waste were identified. Per capita solid waste generation in campus was estimated at $0,052 \mathrm{~kg} /$ person/day for the year

\footnotetext{
* M.Sc. Universidad Pontificia Bolivariana de Bucaramanga (Colombia). luis.castillo@upb.edu.co

** Ph.D. Universidad Pontificia Bolivariana de Bucaramanga (Colombia). marianela.luzardo@upb.edu.co
} 
se hizo una descripción multivariante de la información y con ello se obtuvo un modelo que, además de cumplir con los supuestos estadísticos, explica el comportamiento del $85,9 \%$ de la información.

Palabras clave: Residuos sólidos, Comida compostable, Aprovechamiento de residuos sólidos.
2012. Statistical analysis was performed by a multivariable information description, resulting in a model that fitted to $85,9 \%$ of the data, which adjusted well to the assumed statistical parameters.

Keywords: Solid Waste, Compostable Food, Final Disposition, Solid Waste Reuse and Recycle, Main Analysis Component, Per Capita Generation. 


\section{INTRODUCCIÓN}

La generación de residuos sólidos es inherente a la naturaleza humana, en cuyo diario vivir y en cualquier actividad genera una cantidad de desechos que se debe considerar.

En Colombia, durante el año 2011, se llevaron a disposición final 26.537 toneladas de residuos sólidos [1], mostrando un incremento del $8 \%$ con respecto al año 2010; este dato se hace aún más preocupante si se correlaciona con la tasa de crecimiento poblacional, que para el mismo año fue calculada en $1,18 \%$ [2], pues se demuestra así que el aumento en la producción de residuos no está ligado únicamente al incremento de la población, y que en Colombia ha venido en aumento la producción de residuos por habitante. Esta situación ya había sido anticipada por Collazos, quien planteó años atrás que "A medida que los países aumentan su desarrollo, también aumenta su producción de residuos por habitante" [3].

Con respecto a la legislación colombiana, es posible observar el esfuerzo del Gobierno central, en primera medida, a través del Decreto 1713 de 2002 [4], con el fin de regular la prestación del servicio público de aseo, en sus componentes recolección, trasporte, barrido, tratamiento, aprovechamiento y disposición final; en segunda medida, se reglamentaron los planes de gestión integral de residuos sólidos (PGIRS), por medio de la Resolución 1045 de 2003 [5]. Es precisamente con la implementación de estas normas que se incorpora claramente el concepto de recolección selectiva de residuos en los municipios, de acuerdo con sus posibilidades de aprovechamiento.

Teniendo en cuenta lo expuesto, y con el fin de contribuir a un desarrollo más sostenible, diversas industrias e instituciones de diferente índole han formulado e implementado PGIRS a nivel interno.
La Universidad Pontificia Bolivariana, seccional Bucaramanga, se encuentra ubicada en el municipio de Floridablanca, área metropolitana de Bucaramanga, y cuenta con un campus moderno con un área construida de $35.000 \mathrm{~m}^{2}$ y un área total de $145.559 \mathrm{~m}^{2}$. La institución, en concordancia con las tendencias nacionales e internacionales, y para dar cumplimiento al Decreto 1299 de 2008 [6], estableció en el año 2011 la conformación del área de gestión ambiental, que dentro de sus funciones principales tiene a su cargo la implementación de PGIRS en la institución.

Luego de dos años de iniciada la implementación del PGIRS en la Universidad Pontificia Bolivariana, seccional Bucaramanga, se hace necesario conocer los alcances de las medidas establecidas, con el fin de potencializar las fortalezas y minimizar las debilidades del programa.

El presente trabajo tiene como finalidad evaluar el manejo dado a los residuos sólidos en la Universidad Pontificia Bolivariana, seccional Bucaramanga, desde el año 2011 hasta el primer semestre de 2013.

\section{Metodología}

La evaluación del manejo de los residuos sólidos en la Universidad Pontificia Bolivariana, seccional Bucaramanga, se desarrolló a lo largo de cuatro etapas: 1) Caracterización de los residuos sólidos que van a disposición final, 2) Cuantificación de residuos destinados a aprovechamiento, 3) Evaluación de otros factores asociados a la gestión de los residuos sólidos y 4) Análisis estadístico de la información.

\section{A. Caracterización de los residuos sólidos que van a disposición final}

La caracterización cualitativa y cuantitativa de los residuos que van a disposición final se realizó 
semestralmente, desde el primer semestre de 2011 hasta el primer semestre de 2013. En todos los casos el trabajo se realizó por un mínimo de siete días consecutivos, exceptuando sábados y domingos. El procedimiento consistió en pesar la totalidad de residuos ubicados en el cuarto de almacenamiento temporal y tomar una muestra representativa de entre 40 y $60 \mathrm{~kg}$, utilizando la técnica del cuarteo propuesta por el Centro Panamericano de Ingeniería Sanitaria y Ciencias del Ambiente [7]. Una vez tomada la muestra se procedió a medir la densidad y separar los residuos en las siguientes categorías, según el Reglamento Técnico del sector de Agua Potable y Saneamiento Básico (RAS), título F [8]: (1) comida y jardinería, (2) papel, (3) cartón, (4) polietileno tereftalato (PET), (5) plástico, (6) vidrio, (7) poliestireno expandido (Icopor), (8) metales y (9) otros. El equipo de trabajo estaba compuesto por un docente y un grupo de entre 3 y 5 estudiantes de la asignatura Residuos Sólidos (Facultad de Ingeniería Ambiental), quienes debían hacer la caracterización como parte del componente práctico de la asignatura.

Una vez realizados los pesajes se calculó el porcentaje en peso de cada una de las categorías separadas, y posteriormente el peso total de ellas. Finalmente, utilizando la expresión (1) se calculó la producción per cápita (PPC), teniendo en cuenta el peso de los residuos y el número total personas.

$$
P P C=\frac{P w}{N p}
$$

Donde:

$P P C=$ producción percápitaen $\left(\frac{k g}{\text { persona } \bullet \text { día }}\right)$

$P w=$ Pesodiarioderesiduos en $\left(\frac{\mathrm{kg}}{\text { día }}\right)$

$N p=$ Número de personas

\section{B. Cuantificación de residuos destinados a aprovechamiento}

Los residuos que actualmente se destinan a procesos de aprovechamiento se cuantificaron por pesaje en el sitio de generación directamente o, en su defecto, en el sitio de almacenamiento temporal. Dentro de los residuos que se someten a aprovechamiento se encuentra comida, papel, cartón, metales y plástico.

\section{Evaluación de otros factores asociados a la gestión de los residuos sólidos}

En este punto se evaluaron aspectos relacionados con facilidades con que cuenta la universidad para hacer separación en la fuente, campañas de concientización, cumplimiento de objetivos y metas propuestas.

\section{Análisis estadístico}

El análisis se realizó en dos partes fundamentales: la primera consistió en reducir la dimensionalidad de la matriz de datos a partir de la técnica de análisis de componentes principales (ACP); con la finalidad de evitar redundancias y destacar relaciones en un número de factores que estarán incorrelacionados entre sí y que describen el comportamiento de los datos. Una vez obtenidos los citados componentes se realiza un análisis de regresión lineal múltiple para conocer cuál de las nuevas variables (componentes) influye en la producción total de residuos [9]. Para el análisis se utilizó el paquete estadístico SPSS V21.

\section{Resultados}

\section{A. Caracterización de los residuos sólidos que van a disposición final}

Los residuos que van a disposición final se almacenan temporalmente en un cuarto dispuesto para tal fin, ubicado en un zona cercana a la entrada de la universidad. La 
recolección la hace diariamente, de lunes a sábado, un prestador del servicio público de aseo autorizado, quien es responsable de llevar los residuos al sitio de disposición final del área metropolitana de Bucaramanga, conocido como El Carrasco. En el gráfico 1 se muestra la distribución porcentual en peso de los residuos que son llevados a disposición final durante los tres años de caracterizaciones realizadas.

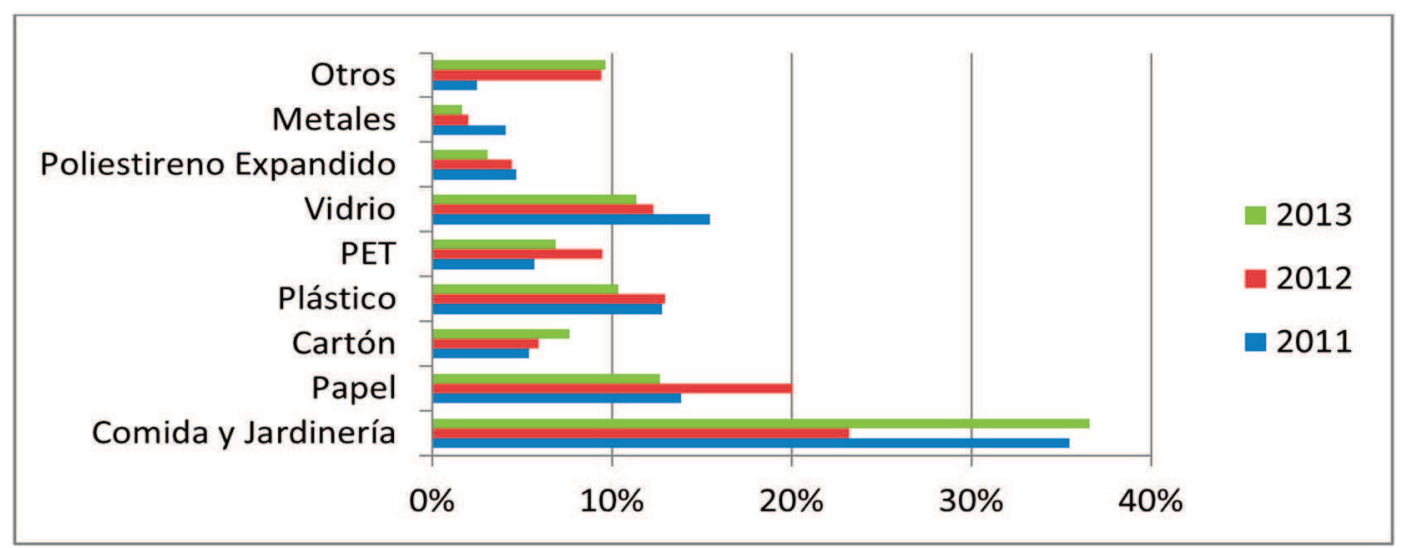

Gráfico 1. Porcentaje de residuos generados en los años 2011 a 2013

Es importante aclarar que dada la gran cantidad de zonas verdes que tiene la universidad, la gran mayoría de residuos de jardinería y poda se destinan a procesos de aprovechamiento o servicios especiales de recolección; sin embargo, aún persisten algunas prácticas inadecuadas dentro del campus, como ubicar parte de estos residuos junto con los residuos ordinarios en el cuarto de almacenamiento temporal; se espera evitar esta situación en un futuro cercano.

Llama la atención el porcentaje de residuos orgánicos biodegradables generados en la institución, los cuales hacen referencia exclusivamente a comida y jardinería; este valor es considerablemente menor si se le compara con el reportado en el Plan de Gestión Integral de Residuos Sólidos para Bucaramanga y su área metropolitana, el cual es del 63\% [10]. Esta situación deja en claro la diferencia en términos de composición de los residuos ordinarios generados en la ciudad (en su mayoría de origen domiciliar) en comparación con los residuos institucionales generados en la universidad. Estudios realizados en universidades de Colombia y en el exterior por Espinosa et al.
[11], Nieto et al. [12], Smyth et al. [13] y Ospina et al. [14] muestran valores de 5\%, 8\%, 22,4\% y $23 \%$, respectivamente, para esta categoría, lo cual demuestra lo variables que pueden llegar a ser estos porcentajes de generación de residuos.

En relación con el papel, se observa una disminución en su parcelación porcentual para el año 2013, lo cual coincide con campañas implementadas desde 2012 para la separación en la fuente de esta categoría a nivel administrativo. La generación de cartón está relacionada con aumento en el uso de materias primas que utilizan cajas de cartón como parte de su embalaje; en este sentido se observa un leve aumento a lo largo del estudio.

Con respecto a las botellas PET, el plástico, el poliestireno expandido y los metales, no se observa un cambio representativo en los porcentajes de generación a lo largo del tiempo.

En la tabla 1 se muestra la generación diaria en kilogramos de cada una de las categorías seleccionadas durante el tiempo de estudio. 
TABla 1

GENERACIÓN DIARIA DE RESIDUOS

\begin{tabular}{|c|c|c|c|}
\hline \multirow{2}{*}{ RESIDUO } & \multicolumn{3}{|c|}{ Cantidad (kg/día) } \\
\hline & 2011 & 2012 & 2013 \\
\hline Comida y jardinería & 52,50 & 68,72 & 149,33 \\
\hline Papel reciclable & & 23,73 & 25,17 \\
\hline Papel no reciclable & & 35,62 & 26,48 \\
\hline Total papel & 20,48 & 59,35 & 51,65 \\
\hline Cartón & 7,99 & 17,63 & 31,26 \\
\hline Plástico & 18,96 & 38,45 & 42,43 \\
\hline PET & 8,46 & 28,00 & 28,07 \\
\hline Vidrio & 22,92 & 36,40 & 46,39 \\
\hline Poliestireno expandido & 6,91 & 13,23 & 12,62 \\
\hline Metales & 6,06 & 6,07 & 6,94 \\
\hline Otros & 3,71 & 27,81 & 39,50 \\
\hline TOTAL & 147,99 & 295,67 & 408,19 \\
\hline
\end{tabular}

La tabla 1 muestra un aumento significativo en la cantidad diaria de residuos a lo largo del tiempo; este incremento coincide con el aumento en el número de personas en la comunidad universitaria a lo largo de mismo periodo, y que se puede ver en la tabla 2 .

\section{TABLA 2}

NÚMERO DE PERSONAS

\begin{tabular}{|c|c|c|c|}
\hline & $\mathbf{2 0 1 1}$ & $\mathbf{2 0 1 2}$ & $\mathbf{2 0 1 3}$ \\
\hline Estudiantes & 5275 & 5602 & 5848 \\
\hline Docentes & 356 & 368 & 377 \\
\hline Administrativos & 203 & 230 & 240 \\
\hline Servicios & 88 & 89 & 127 \\
\hline Total & $\mathbf{5 9 2 2}$ & $\mathbf{6 2 8 9}$ & $\mathbf{6 5 9 2}$ \\
\hline
\end{tabular}

Al hacer un análisis de la cantidad total de residuos generados en el último periodo evaluado (primer semestre de 2013), se puede observar que la totalidad del cartón, del plástico, del PET, del vidrio y de los metales, y el 49,8\% del papel son residuos potencialmente reciclables. Este valor equivale al $44,2 \%$ del total de los residuos generados en un día, lo cual representaría una cantidad diaria en peso de $180,26 \mathrm{~kg}$. Teniendo en cuenta los datos suministrados en las tablas 1 y 2 , es posible establecer la producción per cápita de residuos dada en kg/persona.día.
TABLA 3

PRODUCCIÓN PER CÁPITA DE RESIDUOS

\begin{tabular}{|c|c|c|c|}
\hline \multirow{2}{*}{ RESIDUO } & \multicolumn{3}{|c|}{ PPC (kg/persona/día) } \\
\hline & 2011 & 2012 & 2013 \\
\hline Comida y jardinería & 0,0089 & 0,0109 & 0,0227 \\
\hline Papel & 0,0035 & 0,0094 & 0,0078 \\
\hline Cartón & 0,0013 & 0,0028 & 0,0047 \\
\hline Plástico & 0,0032 & 0,0061 & 0,0064 \\
\hline PET & 0,0014 & 0,0044 & 0,0043 \\
\hline Vidrio & 0,0039 & 0,0058 & 0,0070 \\
\hline Poliestireno expandido & 0,0012 & 0,0021 & 0,0019 \\
\hline Metales & 0,0010 & 0,0010 & 0,0011 \\
\hline Otros & 0,0006 & 0,0044 & 0,0060 \\
\hline TOTAL & 0,025 & 0,047 & 0,062 \\
\hline
\end{tabular}

Como se observa en la tabla 3, la producción per cápita de residuos destinados a disposición final en la Universidad se ha ido incrementando a través de los años, llegando a un total 0,062 $\mathrm{kg} /$ persona.día para el último periodo evaluado. Este aumento puede explicarse por la tendencia que tienen los estudiantes de permanecer cada vez más tiempo dentro de las instalaciones de la universidad, aumentando de esta forma la cantidad de residuos por persona. Otro aspecto influyente está relacionado con el aumento de la población flotante, debido a que en la universidad se realizan semanalmente diferentes actividades, como seminarios, congresos, cursos y otras que conllevan a un aumento representativo de visitantes a la institución.

El aumento más significativo se presenta en la cantidad de residuos de comida generados por persona; factor asociado con el hecho de que cada vez más personas almuerzan dentro de las instalaciones de la institución. Es importante destacar que hasta el primer semestre de 2012 gran parte de estos residuos eran donados a fincas cercanas a la universidad para alimentación porcina; pero esta actividad fue suspendida para dar cumplimiento a la Resolución 2640 de 2007, expedida por el Instituto Colombiano Agropecuario (ICA) [15], la cual dice en su Artículo 14, inciso F, que "Queda prohibido alimentar porcinos con residuos de la 
alimentación humana o con vísceras o carnes de otras especies animales".

También se muestran aumentos importantes en la producción por persona de residuos de cartón, plástico y vidrio. El poliestireno expandido, los metales y el PET permanecen relativamente constantes. Finalmente, una vez más se destaca la eficacia de las campañas de separación en la fuente de papel, pues la cantidad de dicho residuo por persona disminuyó en el último periodo evaluado.

\section{B. Cuantificación de residuos destinados a aprovechamiento}

Dentro de la institución se lleva cabo un proceso de separación en la fuente adelantado principalmente por el departamento de Servicios Generales de la institución, donde se recupera plástico, papel, cartón, metales y residuos de comida compostables, tal como se muestra en la tabla 4.

TABla 4

RESIDUOS RECUPERADOS

\begin{tabular}{|c|c|c|c|}
\hline \multirow{2}{*}{ Residuo } & \multicolumn{3}{|c|}{ Cantidad (kg/año) } \\
\cline { 2 - 4 } & $\mathbf{2 0 1 0}$ & $\mathbf{2 0 1 1}$ & $\mathbf{2 0 1 2}$ \\
\hline Papel & 498 & 1455 & 6596 \\
\hline Cartón & 596 & 751 & 1723 \\
\hline Plástico & 105 & 30 & 153 \\
\hline Metales & 773 & 1247 & 1375 \\
\hline Vidrio & & 10 & 50 \\
\hline Comida & & 5280 & 7656 \\
\hline Total & $\mathbf{1 9 7 2}$ & $\mathbf{8 7 7 3}$ & $\mathbf{9 9 2 6}$ \\
\hline
\end{tabular}

Es importante mencionar que el balance del total de residuos recuperados se hace anualmente, por tanto, no se tienen datos para el año 2013. Se destaca el aumento significativo de todos los residuos aprovechables, especialmente del papel y el cartón, cuyo aumento entre el año 2011 y el año 2012 fue de $345 \%$ y $129 \%$, respectivamente. Todos los residuos recuperados, a excepción de los residuos de comida, son vendidos a cooperativas de recicladores legalmente constituidas y que operan en el área metropolitana de Bucaramanga. Estas ventas han representado importantes ingresos económicos, que se han utilizado para fortalecer los programas de separación en la fuente.

Los residuos de comida compostables se obtienen de las cafeterías, sin incluir ningún tipo de carnes, huesos y comida ya cocinada. Estos residuos recuperados son mezclados con residuos de poda y se llevan a pilas de compostaje ubicadas dentro de las instalaciones de la universidad.

En la tabla 5 se muestran las diferentes categorías de residuos generados, incluyendo residuos recuperados y no recuperados, utilizando como unidad de medida kg/día. Para los residuos recuperados se asumió un año de 44 semanas, y una semana de seis días.

TABLA 5

COMPARACIÓN RESIDUOS RECUPERADOS Y NO RECUPERADOS

\begin{tabular}{|c|c|c|c|c|c|c|}
\hline \multirow{2}{*}{ RESIDUO } & \multicolumn{5}{|c|}{ Cantidad (kg/día) } \\
\cline { 2 - 7 } & \multicolumn{3}{|c|}{$\mathbf{2 0 1 1}$} & \multicolumn{3}{c|}{} \\
\hline & No recuperado & Recuperado & Total & No recuperado & Recuperado & Total \\
\hline Comida y jardinería & 52,50 & & 52,50 & 68,72 & 20 & 88,72 \\
\hline Papel & 20,48 & 5,53 & 26,01 & 59,35 & 24,98 & 84,33 \\
\hline Cartón & 7,99 & 2,84 & 10,83 & 17,63 & 6,53 & 24,16 \\
\hline Plástico & 18,96 & 0,11 & 19,07 & 38,45 & 0,58 & 39,03 \\
\hline PET & 8,46 & & 8,46 & 28,00 & & 28,00 \\
\hline Vidrio & 22,92 & 0,04 & 22,96 & 36,40 & 0,19 & 36,59 \\
\hline Poliestireno Expandido & 6,91 & & 6,91 & 13,23 & & 13,23 \\
\hline Metales & 6,06 & 4,72 & 10,78 & 6,07 & 5,21 & 11,28 \\
\hline Otros & 3,71 & & 3,71 & 27,81 & & 27,81 \\
\hline Total & $\mathbf{1 4 7 , 9 9}$ & $\mathbf{1 3 , 2 4}$ & $\mathbf{1 6 1 , 2 3}$ & $\mathbf{2 9 5 , 6 7}$ & $\mathbf{5 7 , 4 9}$ & $\mathbf{3 5 3 , 1 6}$ \\
\hline
\end{tabular}


Del análisis de los residuos recuperados y no recuperados durante el año 2012 cabe destacar los altos porcentajes de residuos de metales y cartón recuperados: $46,3 \%$ y $27 \%$, respectivamente. En cuanto al papel, se observa un porcentaje de recuperación del 29,6\%; sin embargo, si se descarta el papel no reciclable, como servilletas y papel higiénico, el porcentaje de recuperación de papel es del 48,72\%.

Por otra parte es importante destacar la poca o nula recuperación de plástico y botellas PET, especialmente de estas últimas, aún cuando existe una atractiva oferta de las cooperativas de reciclaje para la compra de este material.

Finalmente, al realizar el cálculo de la producción per cápita total (material recuperado y no recuperado) se obtiene un valor de 0,052 $\mathrm{kg} /$ persona. día para el año 2012; este valor se encuentra por encima del reportado por Nieto et al. para una universidad colombiana, el cual es de $0,023 \mathrm{~kg} /$ persona.día [12], y por debajo del reportado por Espinosa et al. para una universidad internacional en país en vía de desarrollo, donde se obtuvo un valor de $0,110 \mathrm{~kg} /$ persona [11]. Estos resultados muestran que los valores de PPC obtenidos en la institución están dentro de los promedios para universidades en vía de desarrollo.

\section{Evaluación de otros factores asociados a la gestión de los residuos sólidos}

La Institución cuenta en la actualidad con 18 puntos ecológicos distribuidos a lo largo y ancho del campus universitario. Principalmente, los sitos cuentan con tres recipientes de diferentes colores y su respectivo instructivo para hacer la respectiva separación. La definición de los colores utilizados se hizo basándose en las recomendaciones establecidas en la Guía Técnica Colombiana (GTC) 24. De esta forma se tiene un contenedor verde para residuos ordinarios, un contenedor azul para plástico y un contenedor gris para papel [16]. Igualmente, se tienen contenedores blancos, ubicados únicamente en las cafeterías, para disposición de vidrio.

Aun cuando los contenedores fueron adquiridos y ubicados en el segundo semestre de 2011, hasta ahora no ha sido posible tener una adecuada separación en la fuente, ya que se observa una mezcla total de residuos en todos los contenedores sin importar su color. Por otra parte, también se observa que todos los contenedores tienen una bolsa en su interior, dentro de la cual son recolectados los residuos; el color de dichas bolsas, en la mayoría de las ocasiones, no coincide con el color del contenedor, haciendo difícil la identificación de los residuos contenidos en ellas. Infortunadamente, en la actualidad todas las bolsas recolectadas de los puntos ecológicos son llevadas al cuarto de almacenamiento temporal y posteriormente a disposición final sin ningún tipo de aprovechamiento.

Teniendo en cuenta lo anterior, es claro que la comunidad universitaria no tiene un conocimiento claro de la forma como se debe hacer la separación en la fuente de los residuos sólidos; por tanto, los puntos ecológicos no están cumpliendo con su función planeada.

En cuanto al sitio de almacenamiento temporal de residuos, este cumple con todos los requisitos establecidos por la legislación en el Decreto 1140 de 2003 [17], a excepción de su ubicación, ya que el cuarto se encuentra cerca de aulas de clase y cafeterías, lo cual podría causar molestias por olores ofensivos; sin embargo, teniendo en cuenta que los residuos no pasan más de 24 horas en el sitio de almacenamiento temporal, se minimiza en gran medida este inconveniente.

Finalmente, es importante destacar la labor del Área de Gestión Ambiental en pro de la mejora del manejo de los residuos en la institución. Se han implementado campañas exitosas de separación en la fuente de papel y cartón a nivel 
administrativo, y semestralmente se realizan campañas para orientar a toda la comunidad universitaria en cuanto al buen uso de los puntos ecológicos. Igualmente, en 2012 se plantearon metas orientadas a la reducción de la generación de residuos y al aumento del material recuperado. La evaluación del cumplimiento de estas metas se prevé para el final de 2013.

\section{Análisis estadístico}

$\mathrm{Al}$ analizar las correlaciones entre las variables, se aprecia en la tabla 6 que entre las variables regresoras existen valores próximos \pm 1 , indicando que existe una fuerte relación lineal entre ellas. Se destacan en esta situación: el plástico con poliestireno $(0,742)$, el PET con papel recuperado $(0,722)$, plástico recuperado $(0,731)$, metal recuperado $(0,714)$ y vidrio recuperado $(0,705)$. De igual manera se encuentran muy relacionadas: papel con papel recuperado $(0,683)$, plástico recuperado $(0,652)$ metal recuperado $(0,683)$ y vidrio recuperado $(0,690)$; el papel recuperado con plástico recuperado $(0,999)$, metal recuperado $(0,900)$ y vidrio recuperado $(0,925)$; plástico recuperado con metal recuperado $(0,994)$ y vidrio recuperado $(0,997) \mathrm{y}$, por último, metal recuperado y vidrio recuperado $(0,999)$.

TABLA 6

Correlaciones

\begin{tabular}{|c|c|c|c|c|c|c|c|c|c|c|c|c|}
\hline & Plástico & PET & Vidrio & $\begin{array}{c}\text { Comida } \\
\text { y Jardi- } \\
\text { nería }\end{array}$ & \begin{tabular}{|c|} 
Papel \\
y Car- \\
tón \\
\end{tabular} & Metales & $\begin{array}{c}\text { Papel y } \\
\text { Cartón } \\
\text { Recuperado }\end{array}$ & $\begin{array}{l}\text { Residuos de } \\
\text { Comida Re- } \\
\text { cuperados }\end{array}$ & $\begin{array}{c}\text { Plástico } \\
\text { Recuperado }\end{array}$ & $\begin{array}{c}\text { Metales } \\
\text { Recuperados }\end{array}$ & $\begin{array}{c}\text { Vidrio } \\
\text { Recuperado }\end{array}$ & $\begin{array}{c}\text { Poliestireno } \\
\text { Extendido }\end{array}$ \\
\hline Plástico & 1 & & & & & & & & & & & \\
\hline PET & ,470 & 1 & & & & & & & & & & \\
\hline Vidrio & ,441 & ,372 & 1 & & & & & & & & & \\
\hline $\begin{array}{l}\text { Comida y } \\
\text { Jardinería }\end{array}$ & 292 & ,331 & ,482 & 1 & & & & & & & & \\
\hline Papel y Cartón &, 379 & ,558 & ,335 & ,294 & 1 & & & & & & & \\
\hline Metales & ,243 & 161 & ,079 &,- 027 &,- 020 & 1 & & & & & & \\
\hline $\begin{array}{c}\text { Papel y Cartón } \\
\text { Recuperado }\end{array}$ & ,506 & ,722 & ,479 & ,484 & 683 & ,088 & 1 & & & & & \\
\hline $\begin{array}{c}\text { Residuos } \\
\text { de Comida } \\
\text { Recuperados }\end{array}$ &, 256 & 298 & ,361 & ,727 & ,332 & ,101 & ,402 & 1 & & & & \\
\hline $\begin{array}{c}\text { Plástico } \\
\text { Recuperado } \\
\end{array}$ & ,499 & ,731 & ,479 & ,454 & 652 & ,083 & 0,999 & ,409 & 1 & & & \\
\hline $\begin{array}{c}\text { Metales } \\
\text { Recuperado }\end{array}$ &, 502 & ,714 & ,469 & ,464 & 683 & ,081 & 0,900 & ,399 & 0,994 & 1 & & \\
\hline $\begin{array}{c}\text { Vidrio } \\
\text { Recuperado }\end{array}$ &, 510 & ,705 & ,480 &, 500 & 690 & ,079 & 0,925 & ,402 & 0,997 & 0,999 & 1 & \\
\hline $\begin{array}{l}\text { Poliestireno } \\
\text { Expandido }\end{array}$ &, 742 & ,501 &, 326 & , 166 & 411 &, 351 &, 454 & ,144 & ,454 & ,465 & 474 & 1 \\
\hline
\end{tabular}

Al realizar el ACP se obtiene que el porcentaje de variabilidad total explicado para el análisis, usando dos componentes, es de $67,18 \%$, considerándose suficiente para describir la información, tal como se muestra en la tabla 7. 
TABLA 7

VARIANZA TOTAL EXPLICADA

\begin{tabular}{|c|c|c|c|c|c|c|}
\hline \multirow{2}{*}{ Componente } & \multicolumn{5}{|c|}{ Autovalores iniciales } & \multicolumn{3}{c|}{$\begin{array}{c}\text { Sumas de las saturaciones al cuadrado de la } \\
\text { extracción }\end{array}$} \\
\hline & Total & \% de la varianza & \% acumulado & Total & \% de la varianza & \% acumulado \\
\hline 1 & 6,579 & 54,823 & 54,823 & 6,579 & 54,823 & 54,823 \\
\hline 2 & 1,482 & 12,353 & 67,177 & 1,482 & 12,353 & 67,177 \\
\hline 3 & 1,249 & 10,408 & 77,585 & & & \\
\hline 4 &, 787 & 6,562 & 84,147 & & & \\
\hline 5 &, 602 & 5,019 & 89,167 & & & \\
\hline 6 &, 461 & 3,844 & 93,011 & & & \\
\hline 7 &, 393 & 3,277 & 96,287 & & & \\
\hline 8 &, 246 & 2,049 & 98,337 & & & \\
\hline 9 &, 200 & 1,663 & 100,000 & & & \\
\hline 10 & $9,694 \mathrm{E}-9$ & $8,078 \mathrm{E}-8$ & 100,000 & & & \\
\hline 11 & $5,402 \mathrm{E}-16$ & $4,502 \mathrm{E}-15$ & 100,000 & & & \\
\hline 12 & $-9,781 \mathrm{E}-16$ & $-8,151 \mathrm{E}-15$ & 100,000 & & & \\
\hline \multicolumn{7}{|l|}{ Método de extracción: Análisis de Componentes Principales. } \\
\hline
\end{tabular}

Por lo tanto, se tienen dos componentes no correlacionados entre sí. En este orden de ideas, se aprecia en el gráfico 2 que el componente 2 (eje vertical) está definido fundamentalmente por

Gráfico de componentes

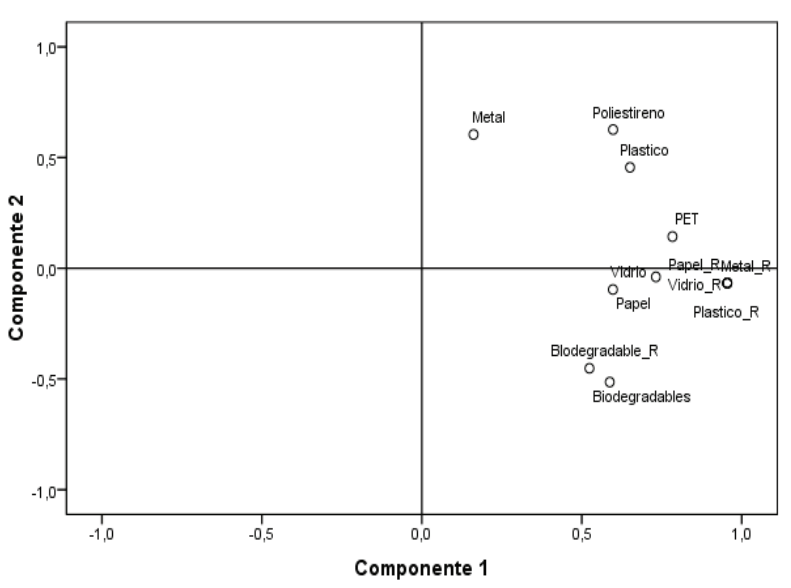

Gráfico 2. Gráfico de Componentes Principales. las variables metal no recuperado y poliestireno expandido; el resto de las variables hacen parte del eje componente 1 , lo que se corrobora en la tabla 8 .

\section{TABLA 8}

MATRIZ DE COMPONENTES

\begin{tabular}{|l|c|c|}
\hline & \multicolumn{2}{|c|}{ Componente } \\
\hline & $\mathrm{C} 1$ & $\mathrm{C} 2$ \\
\hline Plástico &, 651 &, 456 \\
\hline PET &, 783 &, 143 \\
\hline Vidrio &, 597 &,- 096 \\
\hline Comida y jardinería &, 587 &,- 514 \\
\hline Papel &, 732 &,- 039 \\
\hline Metal &, 162 &, 604 \\
\hline Papel_R &, 955 &,- 067 \\
\hline BIodegradable_R &, 524 &,- 452 \\
\hline Plástico_R &, 955 &,- 067 \\
\hline Metal_R &, 955 &,- 067 \\
\hline Vidrio_R &, 955 &,- 067 \\
\hline Poliestireno &, 598 &, 626 \\
\hline \multicolumn{2}{|l|}{ Método de extracción: Análisis de componentes principales. } \\
\hline
\end{tabular}


Ahora bien, los valores de las dos variables "no observables", que serán $\mathrm{C} 1$, para el componente 1, y C2 para el componente 2, se obtienen de la matriz para el cálculo de las puntuaciones en los componentes (Tabla 9), resultando que estas nuevas variables tienen las expresiones (2) y (3).

\section{TABla 9}

Matriz de COEFICIENTES PARA El CÁlCUlo DE LAS PUNTUACIONES EN LAS COMPONENTES

\begin{tabular}{|l|c|c|}
\hline & \multicolumn{2}{|c|}{ Componente } \\
\hline & $\mathrm{C} 1$ & $\mathrm{C} 2$ \\
\hline Plástico &, 099 &, 307 \\
\hline PET &, 119 &, 096 \\
\hline Vidrio &, 091 &,- 065 \\
\hline Bio degradables &, 089 &,- 347 \\
\hline Papel &, 111 &,- 026 \\
\hline Metal &, 025 &, 407 \\
\hline Papel_R &, 145 &,- 045 \\
\hline Bio degradable_R &, 080 &,- 305 \\
\hline Plástico_R &, 145 &,- 045 \\
\hline Metal_R &, 145 &,- 045 \\
\hline Vidrio_R &, 145 &,- 045 \\
\hline Poliestireno &, 091 &, 422 \\
\hline Método de extracción: Análisis de componentes principales. \\
\hline
\end{tabular}

$$
\begin{aligned}
& C_{1}=0,099 \text { Plástico }+0,110 \text { Pet }+0,091 \text { Vidrio }+0,089 \text { biodeg }+0,111 \text { Papel }+0,025 \mathrm{~m} \\
& +0,145 \text { Papel }_{R}+0,08 \text { biodeg }_{R}+0,145\left(\text { Plas }_{R}+\text { metal }_{R}+\text { vidrio }_{R}\right)+0,091 \text { Poliestireno }
\end{aligned}
$$

$C_{2}=0,307$ Plástico + 0,096Pet - 0,065Vidrio - 0,347biodeg - 0,026 Papel +0,407metal -

0,045 Papel $_{R}-0,305$ biodeg $_{R}-0,045\left(\right.$ Plas $_{R}+$ metal $_{R}+$ vidrio $\left._{R}\right)$ 0,422Poliestireno

Con los valores que se obtienen de las expresiones (2) y (3) se hace el análisis de regresión lineal:

De donde

$$
Y i=\beta_{0}+\beta_{1} \cdot C_{1}+\beta_{2} \cdot C_{2}+\varepsilon_{i}
$$

$$
\hat{Y i}=\hat{\beta}_{0}+\hat{\beta}_{1} \cdot C_{1}+\hat{\beta}_{2} \cdot C_{2}
$$

Una vez estimados los coeficientes se tiene el modelo:

$$
\begin{array}{ccc}
\hat{Y} i=263,611+110,775 \cdot C_{1}-19,889 \cdot C_{2} \\
\mathrm{t}=33,94 & \mathrm{t}=13,65 & \mathrm{t}=-2,55 \\
(\mathrm{p}=0,00) & (\mathrm{p}=0,00) & (\mathrm{p}=0,02)
\end{array}
$$


De la expresión (6) se puede deducir que por cada kilo del componente 1 que se incremente, el peso promedio diario se incrementa en 110.775 $\mathrm{kg}$, mientras que por cada kilo del componente 2 que se incremente, el peso promedio diario disminuye en $19.889 \mathrm{~kg}$. En la tabla $10 \mathrm{se}$ muestra el modelo de regresión obtenido en la expresión (6), y el cual explica el $85,9 \%$ de la variabilidad total de los datos.

\section{TABLA 10}

AnÁlisis de Varianza del Modelo de

\begin{tabular}{lccccc}
\multicolumn{7}{c}{ REGRESIÓN } \\
\hline Fuente & SC & gl & CM & F & Sig \\
\hline Regresión & 410518,257 & 2 & 205259,128 & 97,523 &, $000^{\mathrm{a}}$ \\
Residual & 67350,886 & 32 & 2104,715 & & \\
\hline Total & 477869,143 & 34 & & & \\
\hline
\end{tabular}

En cuanto al cumplimiento de los supuestos que se deben verificar en todo modelo de regresión, en primer lugar se tiene el estadístico Durbin Watson $\mathrm{d}=1,93$, que indica la inexistencia de autocorrelación entre los residuos; la prueba de Levene no dio significativa, indicando la presencia de homocedasticidad; con respecto a la multicolinealidad, los estadísticos de tolerancia para cada uno de los componentes es de 0,99 mayor a 0,10, garantizando la no colinealidad entre las nuevas variables por ser

\section{Conclusiones}

El estudio desarrollado en la Universidad Pontificia Bolivariana, seccional Bucaramanga, muestra que el mayor residuo generado en la institución es la categoría correspondiente a comida y jardinería, ya que muestra para el último periodo evaluado una participación del $37 \%$, correspondiente a $149,33 \mathrm{~kg} /$ día.

El promedio actual de residuos en la universidad es de $408,19 \mathrm{~kg} /$ día, de los cuales el $44,2 \%$ es potencialmente aprovechable. Teniendo en cuenta lo anterior, la implementación de un un análisis de regresión proveniente del ACP cuyos componentes no están correlacionados, $\mathrm{y}$, por último, los residuos se distribuyen normal (prueba Kolmogorov-Smirnof resultó 0,759 con $\mathrm{p}=0,612$ ) corroborándose en el gráfico 3 .

\section{Gráfico P-P normal de regresión Residuo tipificado}

Variable dependiente: Peso Total(kg)

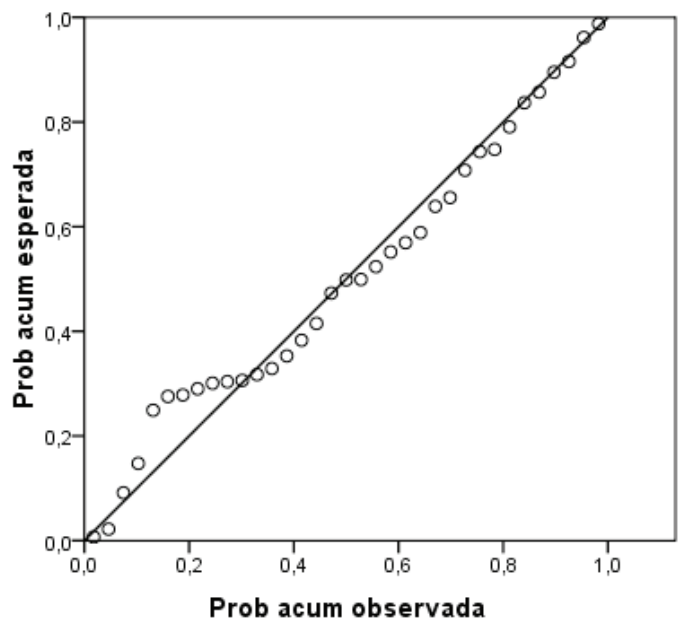

Gráfico 3. Gráfico de Probabilidad Normal

Se concluye que el modelo obtenido para el peso es estadísticamente significativo.

programa adecuado de separación en la fuente evitaría que $180,26 \mathrm{~kg} /$ día de residuos fueran llevados al relleno sanitario.

Se evidenciaron buenas prácticas de aprovechamiento de residuos, destacándose la recuperación del 48,72\% del papel reciclable, el $46,3 \%$ de los metales y $29 \mathrm{~kg} /$ día de residuos compostables. Igualmente, llama la atención el bajo porcentaje de aprovechamiento de plásticos.

Con respecto a la producción per cápita, se observa que para el año 2012 alcanza una cifra de $0,052 \mathrm{~kg} /$ persona.día; este valor está dentro 
de los valores de PPC reportados por la literatura para países en vías de desarrollo.

La variabilidad total de la información fue presentada en dos ejes que la explicaron en un poco más del $67 \%$.

Se obtuvo un modelo estadísticamente significativo para el peso total de residuos, el cual cumple con todos los supuestos estadísticos.

Las campañas adoptadas por el Área de Gestión Ambiental en la universidad han dado buenos resultados para la separación en la fuente a nivel administrativo; sin embargo, no ha sido posible tener el mismo éxito en toda la comunidad universitaria. Esto se evidencia en los puntos ecológicos distribuidos en el campus, donde predomina la mezcla de residuos en todos los contenedores.

\section{REFERENCIAS}

[1] República de Colombia, Superintendencia de Servicios Públicos Domiciliarios -SSPD-. Situación de la disposición final de residuos sólidos en Colombia-Diagnóstico 2011. 26 p.

[2] DANE. El Censo 2005 deja ver una realidad demográfica. Comunicado de Prensa. 2005.

[3] H. Collazos, R. Duque, Asociación Colombiana de Ingeniería Sanitaria y Ambiental. ACODAL. 5. ${ }^{a}$ Edición. Santafé de Bogotá. Acodal, 1998. $170 \mathrm{p}$.

[4] República de Colombia, Ministerio de Desarrollo Económico. Decreto 1713 de 2002 de agosto 6, por el cual se reglamenta la Ley 142 de 1994, la Ley 632 de 2000 y la Ley 689 de 2001, en relación con la prestación del servicio público de aseo, y el Decreto Ley 2811 de 1974 y la Ley 99 de 1993 en relación con la Gestión Integral de Residuos Sólidos.
[5] República de Colombia, Ministerio de Ambiente, Vivienda y Desarrollo Territorial. Resolución 1045 de 2003 de septiembre 26, por la cual se adopta la metodología para la elaboración de los Planes de Gestión Integral de Residuos Sólidos, PGIRS, y se toman otras determinaciones.

[6] República de Colombia, Ministerio de Ambiente, Vivienda y Desarrollo Territorial. Decreto 1299 de 2008 de abril 22, por el cual se reglamenta el departamento de gestión ambiental de las empresas a nivel industrial y se dictan otras disposiciones.

[7] Hojas de divulgación técnica. Procedimientos estadísticos para los estudios de caracterización de residuos sólidos. Centro Panamericano de Ingeniería Sanitaria y Ciencias del Ambiente. ISSN: 1018-5119. Junio 2005.

[8] República de Colombia. Ministerio de Desarrollo Económico. Dirección de Agua Potable y Saneamiento Básico. Reglamento técnico del sector de agua potable $\mathrm{y}$ saneamiento básico RAS-2000. Sistemas de Aseo Urbano. Bogotá D.C., Noviembre de 2000. 224p.

[9] C. Chatfield and A. J. Collins, Introduction to Multivariate Analysis. Chapman and Hall, London, 1980.

[10] Universidad Industrial de Santander. Plan de Gestión Integral de Residuos Sólidos del Área Metropolitana de Bucaramanga. Diagnóstico Técnico y Operativo. 144 p.

[11] R. M. Espinosa et ál. "Integral urban solid waste management program in a Mexican university". ELSEVIER, Waste Management 28 (2008) S27-S32.

[12] O. Nieto et ál., "Diagnosis of Generations and Handling of Solid Residuals in the University of Quindío". Rev. Invest. Univ. Quindio (20): 153-165. Junio 2010. 
[13] D. Smyth, A. Fredeen, A. Booth, "Reducing solid waste in higher education: The first step towards 'greening' a university campus". ELSEVIER. Resources, Conservation and Recycling 54 (2010): 1007-1016.

[14] A. Ospina, O. Bernal, A. Chávez, "Characterization Phase of Non-Hazardous Solid Waste in Environmental Management Institutional Plan of UMNG". Revista Academia y Virtualidad. Volumen 3, N. ${ }^{\circ} 1$. ISSN 2011-0731. 2010.

[15] Instituto Colombiano Agropecuario ICA. Resolución N. ${ }^{\circ} 002640$ de septiembre 28 de 2007, por la cual se reglamentan las condiciones sanitarias y de inocuidad en la producción primaria de ganado porcino destinado al sacrificio para consumo humano. $20 \mathrm{p}$.

[16] ICONTEC. Norma Técnica Colombiana GTC 24. Gestión Ambiental. Residuos Sólidos. Guía para la separación en la fuente. Mayo 20 de 2009.18 p.

[17] República de Colombia, Ministerio de Ambiente, Vivienda y Desarrollo Territorial. Decreto 1140 de 2003 de mayo 7, por el cual se modifica parcialmente el Decreto 1713 de 2002, en relación con el tema de las unidades de almacenamiento, y se dictan otras disposiciones. 